\title{
REALIDAD AUMENTADA, VIDEOJUEGOS Y CAMBIO CLIMÁTICO
}

\section{AUGMENTED REALITY, VIDEOGAMES AND CLIMATE CHANGE}

\author{
Ramón Fabregat ${ }^{1 *}$, Hendrys F. Tobar ${ }^{2}$, Silvia M. Baldiris ${ }^{3}$, Jorge Hernández ${ }^{4}$ \\ Recibido para publicación: 22 febrero de 2013- Aceptado para publicación: 24 de abril del 2014
}

\section{RESUMEN}

En este documento se presentan la Realidad Aumentada (RA) y los videojuegos como dos tecnologías de tendencia actual que pueden ser aplicadas (de manera independiente y en conjunción) hacia procesos relacionados con el cambio climático. El artículo se centra en la aplicación de estas tecnologías principalmente a procesos de enseñanza-aprendizaje, sensibilización y concienciación sobre el cambio climático. Por un lado se presenta la realidad aumentada y algunos ejemplos de aplicaciones existentes relacionadas con el cambio climático. Por otro lado, se presentan los videojuegos como herramientas útiles para el aprendizaje, a través de la filosofía del aprendizaje basado en juegos. Se discuten algunos principios del aprendizaje basado en juegos y se muestran algunos ejemplos de los videojuegos aplicados al cambio climático. Por último se presenta los videojuegos con realidad aumentada y se propone una clasificación de estos. Se concluye que los videojuegos y la Realidad Aumentada son dos tecnologías que actualmente deben ser utilizadas para procesos relacionados con la educación sobre el cambio climático debido a sus características y naturaleza.

Palabras Clave: Realidad Aumentada, Videojuegos, Cambio Climático, Aprendizaje

PALABRAS CLAVES: Sistemas Recomendadores, educación, comercio electrónico, orientación vocacional, información.

\footnotetext{
${ }^{1}$ Doctor en Ingenieria Industrial, Profesor Titular de Universidad, Universitat de Girona, C/ Emili Grahit, 91,17003, Girona, Cataluña, España. ramon.fabregat@udg.edu, + 34972418484

2 Estudiante de Doctorado en Tecnología, Universitat de Girona, Girona, Cataluña, España.

3 Doctora en Tecnología, Investigadora, Universitat de Girona, Girona,, España, sbaldiris@gmail.com, + 34972418484

${ }^{4}$ Ingeniero de Sistemas, Profesor, CUC, jhernandez@cuc.edu.co
} 
Ramón Fabregat, Realidad Aumentada

\begin{abstract}
In this paper, Augmented Reality (AR) and Videogames are presented as two technologies suitable to be applied (independently and mixed) to Climate Change issues. The article is centered on teaching learning, sensitization and awareness campaigns about Climate Change. On one hand, Augmented Reality and some examples of Climate-Change-related applications are presented. On the other hand, videogames are presented as useful tools for learning under the philosophy of Digital Game-Based Learning (DGBL). Some principles of DGBL are discussed and some examples of videogames applied to Climate Change issues are presented. Lastly Augmented Reality games are explored and an early classification is introduced. Finally, it is concluded that videogames and Augmented Reality are two current technologies that must be applied to processes related to education on climate change due to their features and nature.
\end{abstract}

Keywords: Augmented Reality, Videogames, Climate Change, Learning

\section{INTRODUCCIÓN}

Existen dos tecnologías emergentes, la Realidad Aumentada y los Videojuegos, que pueden ser aplicadas en diversos entornos entre los que se encuentran los relacionados con el Cambio Climático. Por un lado, en las aplicaciones de Realidad Aumentada se mezcla contenido del mundo real con elementos virtuales. Por otro lado, los Videojuegos son aplicaciones de entretenimiento diseñadas para interactuar fuertemente con un usuario y en ciertas ocasiones con unos objetivos que van más allá del de entretener.

Consideramos que estas tecnologías, cada vez más fácilmente accesibles al público en general, son parte de una revolución informática que permitirá dar a conocer mejor los aspectos del Cambio Climático y con ello permitir una aumentar la concientización de la sociedad en general sobre esta problemática.

Actualmente, en el campo del Cambio Climático, ya existen aplicaciones orientadas a la divulgación, la publicidad, la educación y la formación e investigación relacionadas con el Cambio Climático realizadas con Realidad Aumentada o Videojuegos.

En este artículo, explicamos las características de estas tecnologías, sus potencialidades tanto cuando son usadas independientemente como cuando son usadas en conjunto y proveemos algunos ejemplos de aplicaciones ya existentes para que además de ilustrar como se pueden utilizar estas tecnologías en este ámbito, intentar inspirar el desarrollo de nuevos aplicaciones 0 proyectos con características similares.

\section{REALIDAD AUMENTADA}

Un sistema de Realidad Aumentada es un sistema que permite la visualización de una capa virtual superpuesta sobre elementos del mundo real (Azuma 1997). En ese sentido,

"aumentar" implica lograr que el ser humano pueda: captar situaciones, detalles, características e información que sus sentidos no podrían captar sin la ayuda de la tecnología o aumentar las capacidades (reales) de un usuario al interactuar con un sistema informático.

Según Azuma (Azuma 1997) para que un sistema se clasifique como sistema de realidad aumentada, este:

- Combina lo real y lo virtual. La información digital es combinada con la realidad.

- Funciona en tiempo real. La combinación de lo real y lo virtual se hace en tiempo real.

- Registra en tres dimensiones. En general la información aumentada se localiza o "registra" en el espacio. Para conservar la ilusión de ubicación real y virtual, ésta última tiende a conservar su ubicación o a moverse respecto a un punto de referencia en el mundo real. 


\subsection{Tipos de Realidad Aumentada basada en la interacción y el tipo de visualización}

En la llustración 1, tomada de (Tobar et al. 2013) se muestran algunos ejemplos de Realidad Aumentada clasificada según la forma en que el usuario interactúa con ella:

- Headworn (Usado en la cabeza): El usuario lleva el dispositivo en la cabeza.

- Hand-held (Sostenido en la mano): El usuario usa un dispositivo portable en la mano.

- Spatial (Espacial): El sistema observa al usuario en un espacio desde una posición fija.

Así mismo, la figura clasifica por tipo de display:

- Video: El resultado de la mezcla de imágenes del mundo real y elementos virtuales se presenta por medio de un video.

- Optical See-Through: Los elementos virtuales se superponen en un medio que permite ver a través de él (como un cristal).

- Projective (proyectiva): Los elementos virtuales se proyectan sobre los objetos del mundo real.

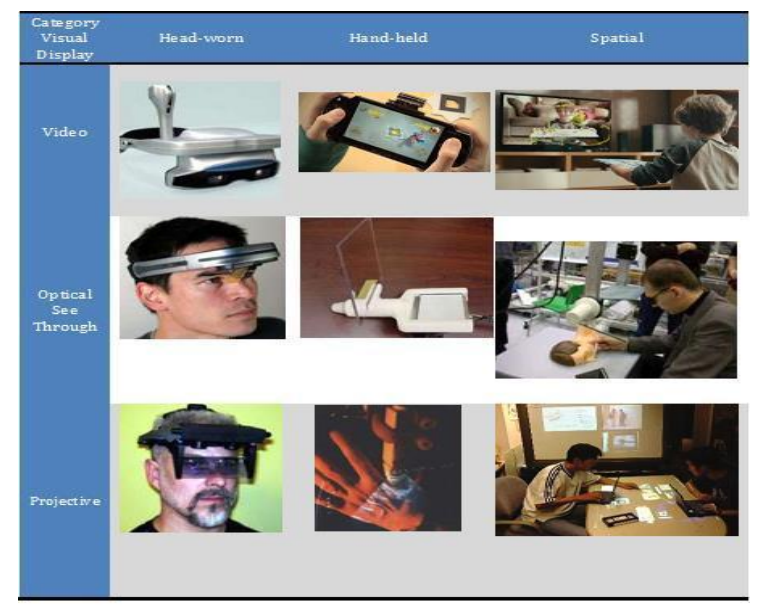

Figura 1. Clasificación de aplicaciones de realidad aumentada (Tobar et al. 2013).

\subsection{Ejemplos de aplicaciones de Realidad Aumentada}

En cuanto al cambio climático, algunos esfuerzos con aplicaciones de realidad aumentada ya se han hecho. Describimos aquí algunos de éstos con el ánimo de mostrar las potencialidades de esta tecnología e inspirar nuevos proyectos a partir de esta revisión.

- El investigador Sean Michael White desarrolló una aplicación para identificar plantas por medio del reconocimiento de imágenes y realidad aumentada. La aplicación se distribuye para dispositivos como el IPhone y permite a los botánicos ahorrar tiempo en búsquedas manuales a partir de fotografías (Fora.tv 2008). La aplicación utiliza unas gafas para visualizar las plantas en tiempo real (llustración 2 (1) )

- El mismo investigador también trabajó en una aplicación para visualización de niveles de dióxido de carbono a partir de datos de un sensor geo-codificado. La aplicación permite ver los niveles del gas de manera visual (llustración 2 (2)) (Kalkofen et al. 2011)

- Según Camerón (Cameron 2010), White habría trabajado en una aplicación para descubrir espacios óptimos para localización de generadores eólicos.

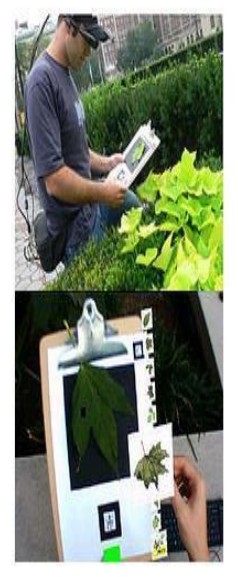

(1)

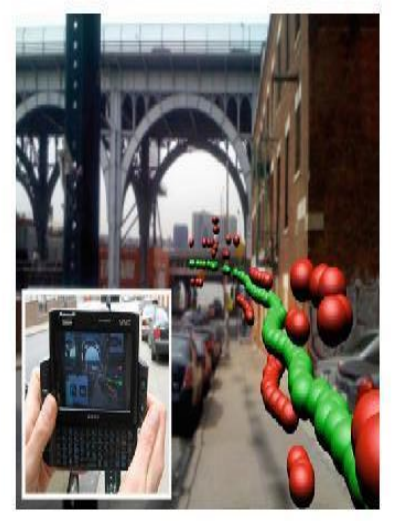

(2)
Figura 2. Ejemplo de aplicaciones ambientales con RA

- Una de las corporaciones más grandes del mundo: General Electric (GE) ha

desarrollado una aplicación interactiva con realidad aumentada que explica los beneficios de usar SmartGrid. En esta 
aplicación, el usuario puede utilizar un marcador para observar una instalación eólica y soplar (hacia el micrófono) para activarla.

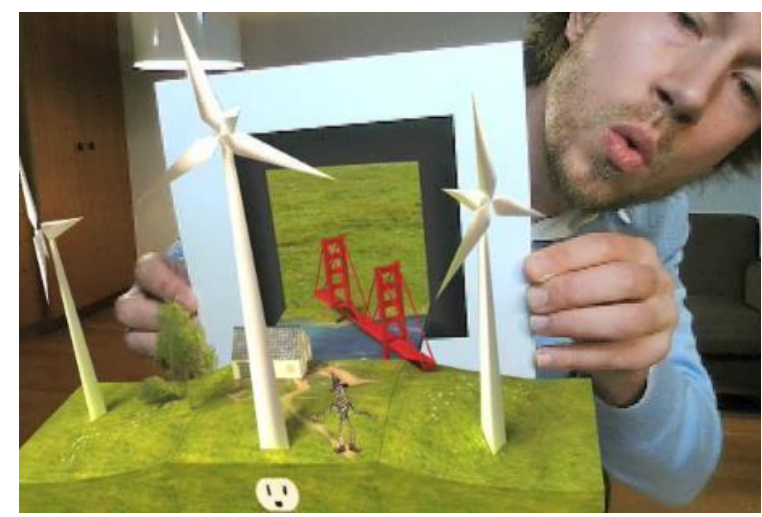

Figura 3. Aplicación de GE con RA para explicar instalaciones eólicas y SmartGrid.

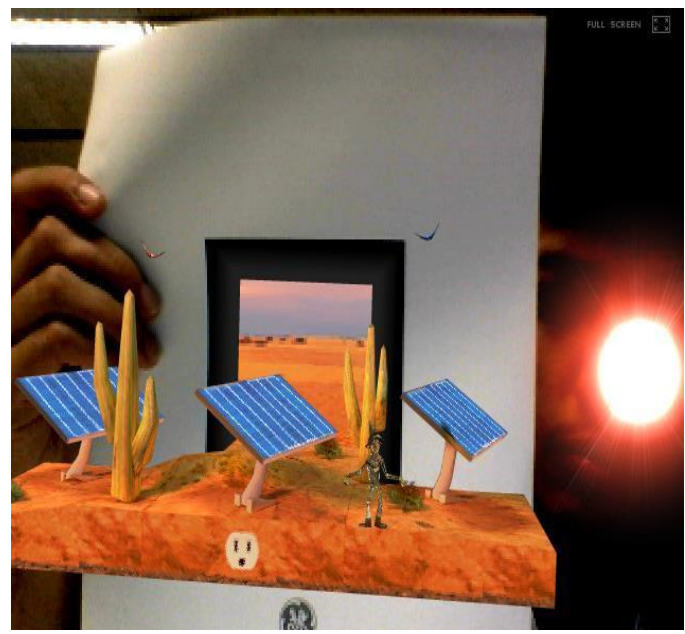

Figura 4. Aplicación de GE para mostrar el funcionamiento de un generador fotovoltaico.

- El "Observatori de Canvi Climàtic" de Valencia creó la iniciativa Valencia 2050. Una experiencia interactiva para observar el cambio climático. En ella, los asistentes pueden interactuar con la aplicación a través de unas tarjetas sobre las que se aplica realidad aumentada proyectiva y con las cuales se puede tener una experiencia para conocer más sobre las emisiones de gas carbónico (llustración 4).

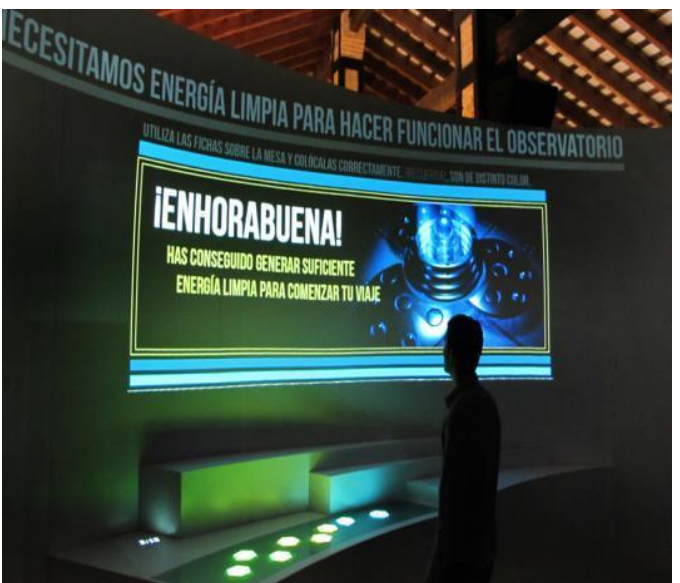

Figura 5. Instalación de "Valencia 2050"

Futuroscope: Una herramienta para ver hacia el futuro. ¿Cómo evolucionarán los animales dependiendo del clima y el medio ambiente? Esta herramienta usa un dispositivo similar a unos binoculares, el cual puede explorar una instalación real con elementos virtuales que representan animales y plantas evolucionando de acuerdo al cambio climático.

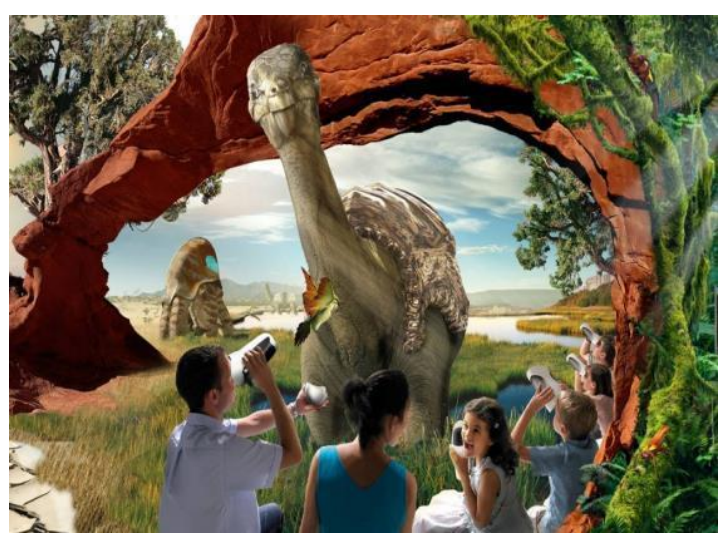

Figura 6. Futuroscope

- La "Fundación Biodiversidad" propone una aplicación para experiencias interactivas familiares sobre el medio ambiente usando RA (llustración 6). El objetivo de esta aplicación es utilizar unos marcadores de realidad aumentada que muestran elementos propios de una ciudad (como carros, industrias, etc.) para ubicarlos adecuadamente de tal manera que se creen dinámicas y relaciones sostenibles en la ciudad virtual. 


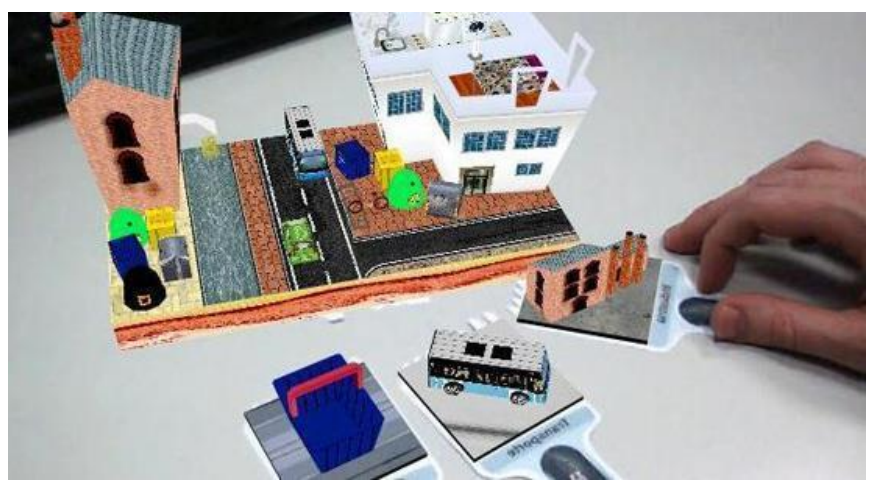

figura 7. Experiencias Interactivas de la Fundación Biodiversidad.

\subsection{Ventajas de la Realidad Aumentada en procesos de enseñanza-aprendizaje}

La realidad aumentada es una tecnología factible de aplicar en distintos ámbitos. Uno de esos ámbitos es la educación. Creemos que dadas las características de la realidad aumentada y su nivel actual de desarrollo y divulgación, la realidad aumentada desempeñará un papel muy importante en procesos que involucren la formación, el aprendizaje y la enseñanza.

La realidad aumentada es útil pues, de acuerdo con el Reporte Horizon 2010 (Johnson et al. n.d.), un reporte anual que se realiza para observar las tecnologías que se aplicarán en la educación, las tecnologías de realidad aumentada deberían estarse aplicando en un lapso de dos a tres años, es decir, hoy en día.

El reporte Horizon afirma, y nosotros estamos de acuerdo, que la realidad aumentada cuenta con fuertes potenciales para proveer experiencias de aprendizaje in situ, así como para obtener aprendizaje a partir de la exploración y el descubrimiento por parte del aprendiz (New Media Consortium, 2010, p 22). Por ello, si bien la tecnología de realidad aumentada tiene diferentes posibles aplicaciones que van desde el ámbito de investigación, hasta la publicidad, creemos que pueden servir para la formación y la educación en "temáticas" relacionadas con el Cambio Climático.

Algunas de las ventajas de la realidad aumentada por las que creemos que los esfuerzos relacionados con el cambio climático deberían centrarse en la formación y educación son:

- Posibilidad de aprendizaje en el lugar donde ocurra (New Media Consortium 2010): La realidad aumentada permite que el estudiante observe objetos del mundo real y que permita ver su interior de manera segura a través de aplicaciones que así lo permitan.

- Aprendizaje experimental sin desplazar al estudiante (Adelsberger et al. 2008): La realidad aumentada es, por su naturaleza, controlada por el usuario. De esa manera, la realidad se puede experimentar de una manera más relevante, lo cual es muy importante para el aprendizaje.

- Participación que involucra al observador (Woods et al. 2004): Por ejemplo, en algunos museos la realidad aumentada ha sido aplicada para que el usuario no sólo sea un observador pasivo, sino que, a partir de la experiencia propia, se involucre con el objeto observado para aprender más de él.

- Aprendizaje Móvil (FitzGerald et al. 2012): El avance de las tecnologías móviles posibilita que el acceso a recursos de realidad aumentada sea más fácil y para un público más amplio.

En síntesis, la realidad aumentada permite un nuevo tipo de aprendizaje mejorado con tecnología y es evidente que puede ser utilizada para ayudar en los procesos de formación, sensibilización y concienciación a la problemática del Cambio Climático.

\section{VIDEOJUEGOS}

Los Videojuegos son aplicaciones software altamente interactivas en las que una experiencia lúdica se lleva a cabo en un dispositivo informático. Los Videojuegos son muy famosos hoy en día, no obstante, no todo el mundo entiende que ellos pueden ser aplicados para objetivos más allá del mero entretenimiento. En este sentido, existen los "Juegos Serios" (Serious Games en inglés). Este tipo de videojuegos incorporan las características lúdicas y llamativas de 
cualquier videojuego, pero su contenido (mecánica, plataforma social, etc.) contribuyen a alcanzar un objetivo superior. Por ejemplo: La educación, la salud, el entrenamiento o la divulgación de campañas de concienciación, como podría ser el caso de la concienciación sobre el Cambio Climático.

En nuestro grupo de investigación hemos trabajado en la aplicación de videojuegos para el aprendizaje y la concienciación. Es de mencionar que la construcción y diseño de estos juegos difieren en aspectos fundamentales con el diseño y construcción de juegos de entretenimiento (Barnes \& Encarnação 2009). Esto es, que los juegos serios se deben pensar primero en su objetivo de aprendizaje 0 concienciación para posteriormente centrarse en proveer una experiencia al jugador que sea relevante para sus necesidades.

A continuación, presentamos algunas de las características de los videojuegos que pueden aportar a las campañas y procesos de divulgación sobre el Cambio Climático.

\subsection{Aprendizaje Basado en Juegos Digitales.}

Desde hace ya algunos años existe una nueva tendencia entre los educadores del mundo denominada "Aprendizaje Basado en Juegos Digitales" (DGBL por sus siglas en inglés) definido por Marc Prensky continuando su obra "Digital Game Based Learning" (Prensky 2001a).

"Si un niño puede aprenderse los nombres de 500 Pokémon, seguramente aprenderá las capitales del mundo." Mark Prensky (Prensky 2001b)

Esta frase resume en cierta forma lo que aquellos estudiosos de este tema argumentan que el aprendizaje que ocurre durante una sesión de juego puede ser asimilado por el jugador/estudiante para que pueda ser aplicado en contextos de la vida diaria. La tendencia del "Aprendizaje Basado en Juegos Digitales" está pensada para ser aplicada en los "Nativos Digitales", es decir los estudiantes que han crecido con las nuevas tecnologías (Prensky 2001b). Sin embargo, sus principios pueden ser aplicados en multitud de contextos para con distintas personas.

Algunos principios por los que el aprendizaje basado en juegos funciona, según James Paul Gee (Gee 2005) son:

1. Identidad: El aprendiz se compromete con el juego.

2. Interacción: Los juegos permiten interactuar, los libros tradicionales no.

3. Producción: Los jugadores no solo consumen juegos, los "producen" jugando y tomando acciones en ellos.

4. Asumir riesgos: Los juegos permiten a los jugadores fallar, pero con pocas consecuencias, algo que no se puede hacer en la vida real.

5. Personalización: Generalmente, un jugador personaliza su juego, lo hace "suyo".

6. Agencia: El sentido de control. Según Gee, los videojuegos permiten una verdadera sensación de propiedad, de control sobre el mundo que les rodea, algo que las escuelas rara vez ofrecen.

7. Problemas bien ordenados: Los juegos le dan constantemente al jugador "conflictos cognitivos" que el jugador aborda, con las herramientas de las que dispone, para alcanzar soluciones y aprendizaje.

La obra de Gee explora otros aspectos que invitan a reconocer a los videojuegos como excelentes herramientas para el aprendizaje. Dentro de estos aspectos encontramos: el reto, el pensamiento sistemático y el valorar el rendimiento antes que la competencia.

Por estas razones, los videojuegos ya han sido aplicados extensamente en el área del aprendizaje. $Y$ por este motivo creemos también que los Videojuegos pueden ser aplicados en campañas de concientización pues su característica de inmersión permitirá un aprendizaje a más largo plazo.

Existen diferentes juegos serios que tienen que ver con el Cambio Climático, estos son algunos ejemplos:

- The Climate Trail: Un juego similar a "Oregon Trail". En "Oregon Trail”, el jugador debe tomar decisiones para salvaguardar a su familia de depredadores $y$ enfermedades. Pero en este juego, el jugador debe tomar decisiones que 
afectarán el clima de su ciudad (Ilustración 8).

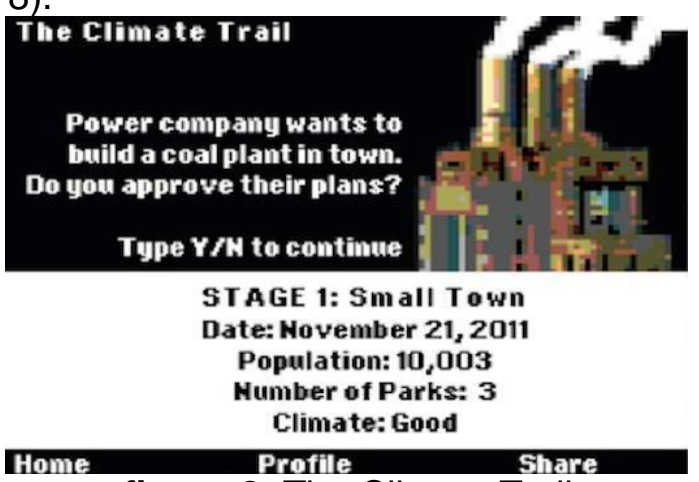

figura 8. The Climate Trail

- Realitree: Un árbol virtual en medio de la ciudad que refleja el estado del clima. La gente lo puede salvar usando sus móviles para reportar como ayudan al clima (llustración 9).

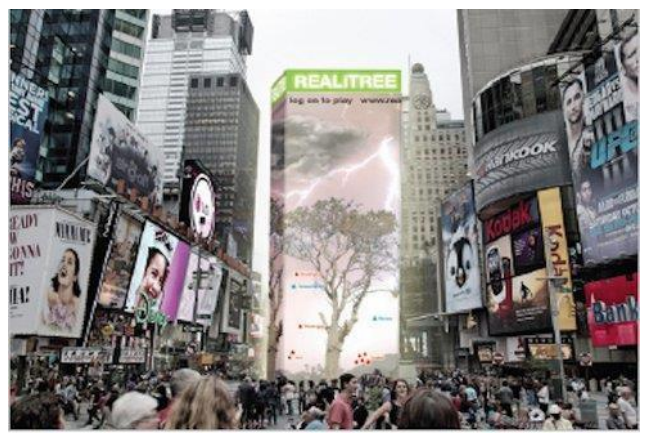

figura 9. The Realitree

- CEO2 : La WWF (World Wild Foundation) creó un juego en el que se simula ser el CEO de una compañía multinacional que debe tener ganancias pero evitar la huella de carbono (llustración 10).

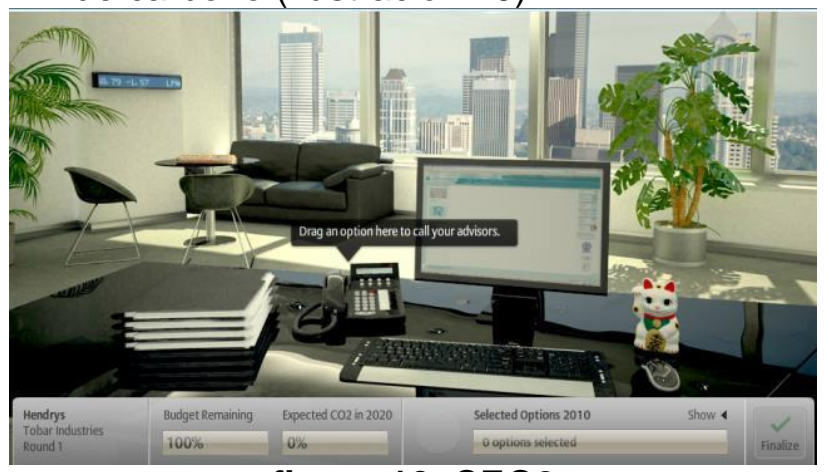

figura 10. CEO2

- HerAdventure: Una súper héroe que debe resolver problemas sociales relacionados con el clima (llustración 11).

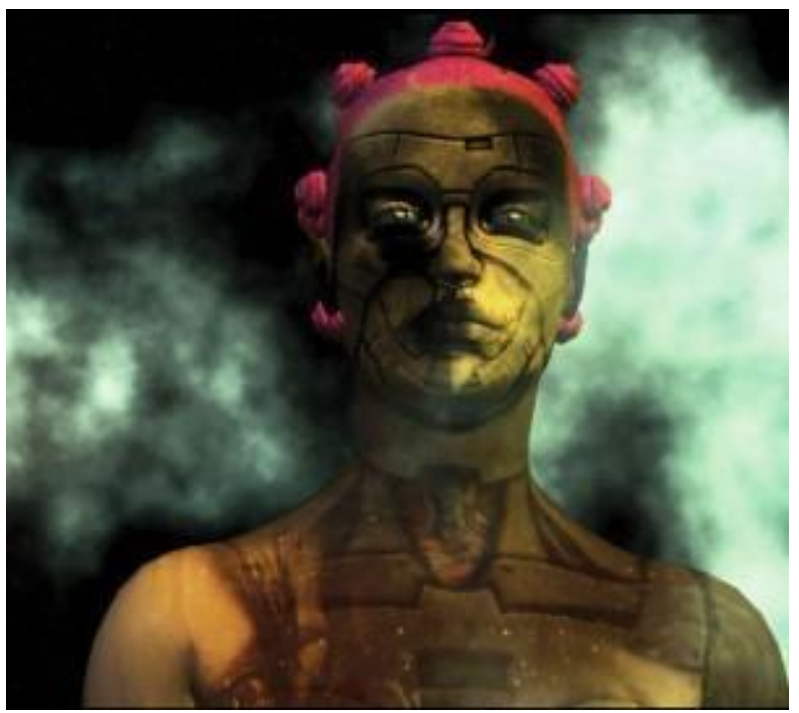

figura 11. HerAdventure

Como vemos, los videojuegos son una tecnología que ya está siendo usada y que dadas sus características tiene un gran potencial de ser aplicada especialmente para divulgar y concienciar a las nuevas generaciones sobre la problemática del Cambio Climático.

\subsection{Videojuegos con Realidad Aumentada}

Una de las aplicaciones fundamentales de la realidad aumentada, son los videojuegos (Krevelen \& Poelman 2010). En los videojuegos con realidad aumentada el usuario interactúa con el juego y sus reglas haciendo uso de los distintos tipos de realidad aumentada que hay.

Generalmente, los videojuegos de realidad aumentada ofrecen al jugador una experiencia diferente, por cuanto hacen uso de las técnicas de rastreo que otros tipos de videojuegos no poseen. El rastreo es una de las características más importantes de una aplicación de realidad aumentada y se refiere a la forma en la que el dispositivo sigue el comportamiento del usuario para que el sistema se actualice de acuerdo a ese comportamiento (lo cual se denomina

"registrar") (Azuma et al. 2001). En este contexto, un buen registro existe cuando los elementos virtuales se alinean adecuadamente con los objetos reales, dando efectivamente, una sensación de "aumento". Por ello, podemos clasificar los tipos de videojuegos 
con Realidad Aumentada según su tipo de rastreo(Tobar et al. 2013) (llustración 2). En la llustración 12 se muestran esquemas para los siguientes tipos de rastreo:

- Videojuego Móvil basado en marcadores: El jugador apunta su dispositivo móvil a un conjunto de marcadores predefinidos y los elementos virtuales del juego aparecen en el video tomado por la cámara del dispositivo (llustración 12 (1)).

- Videojuego Móvil rastreado por el acelerómetro y/o el giroscopio: El dispositivo usa el acelerómetro y/o el giroscopio para registrar los movimientos del usuario y reaccionar ante ello. Para eso se puede usar un dispositivo móvil o existen otros tipos de hardware más complejos (Ilustración 12 (2)).

- Videojuego rastreado por el GPS: La posición del jugador se obtiene a través del dispositivo GPS de su hardware (llustración 12 (3)).

- Videojuego espacial: El jugador se sitúa en frente de un monitor e interactúa con él usando marcadores o dispositivos especiales que se aumentan en la pantalla (llustración 12(4))

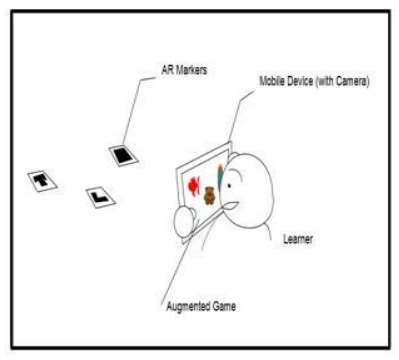

(1) Videowuego móvil basado en marcadores

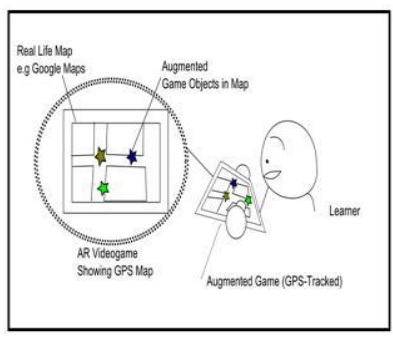

(3) Juegorastreado por GPS
(2) Juego rastreado por acelerómetro o Giroscopio

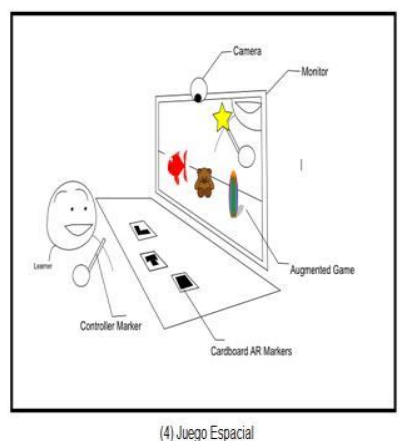

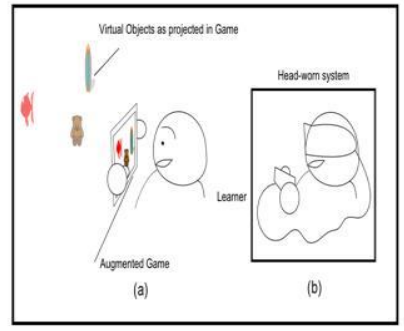

Ilustración 12. Tipos de aplicaciones de realidad aumentada según rastreo
Actualmente existen varios ejemplos de juegos de realidad aumentada en el mercado. Sin embargo, videojuegos orientados al problema del Cambio Climático aún son escasos. Por eso, consideramos que la mezcla de estas dos tecnologías, aunadas a un importante objetivo como este, no sólo es posible, sino que es viable para las iniciativas que apunten a realizar divulgación y educación de esta problemática.

\section{CONCLUSIONES}

Los sistemas recomendadores son una herramienta de apoyo muy importante para tomar decisiones en cualquier campo, por ende su desarrollo debe ser un proceso minucioso que defina muy bien qué tipo de información requerirá del usuario $y / 0$ qué tipo de información tendrá sobre los ítems a recomendar, para que la calidad de la recomendación satisfaga en la mayor medida posible la necesidad del usuario y se vean llenadas sus expectativas.

En el desarrollo del sistema recomendador Student-R se utilizaron tecnologías asequibles y confiables en la construcción de este tipo de sistemas como JAVA, XML y AJAX; el sistema se convierte en una muy buena herramienta para ir probando las ventajas de este tipo de software en el campo educativo.

Se reconoce la importancia del desarrollo de una buena interfaz gráfica de usuario que permita una sencilla interacción de los usuarios con el sistema permitiendo que los resultados del proceso de recomendación se le den a conocer de una forma clara y convincente.

\section{REFERENCIAS}

[1]. Adelsberger, H.H., Kinshuk, Pawlowsky, J.M. \& Sampson, D. 2008. Handbook on Information Technologies for Education and 
Training P. Bernus et al., eds., Springer.

[2]. Azuma, R., Baillot, Y., Behringer, R., Feiner, S., Julier, S. \& Maclntyre, B. 2001. Recent advances in augmented reality. IEEE Computer Graphics and Applications (December): 34-47. (en linea)

http://ieeexplore.ieee.org/xpls/abs_all.js p?arnumber=963459. Acceso: 14 May (2013).

[3]. Azuma, R.T. 1997. A survey of Augmented Reality. PRESENCE: Teleoperators and Virtual Environments.

[4]. Barnes, T. \& Encarnação, L.M. 2009. Serious games. Computer Graphics and... (en linea) http://ieeexplore.ieee.org/xpls/abs_all.js p?arnumber $=4797512$.

[5]. Cameron, C. 2010. Can Augmented Reality Help Save the Planet? ReadWrite. (en linea) http://readwrite.com/2010/06/23/can_a ugmented_reality_help_save_the_plan et.

[6]. FitzGerald, E., Adams, A., Ferguson, R. \& Gaved, M. 2012. Augmented reality and mobile learning: the state of the art. (en linea) http://oro.open.ac.uk/34281/. Acceso: 20 March (2013).

[7]. Fora.tv 2008. Sean White - Demos Tangible Augmented Reality. (en linea) http://www.dailymotion.com/video/xgjkr d_sean-white-demos-tangibleaugmented-reality_news. .
[8]. Gee, J. 2005. Good video games and good learning. Phi Kappa Phi Forum: 33-37. (en linea) http://www.citeulike.org/group/6270/pa ge/2. Acceso: 4 April (2013).

[9]. Johnson, L., Levine, A. \& Smith, R. The 2010 Horizon Report. New Media Consortium. (en linea) http://eric.ed.gov/ERICWebPortal/recor dDetail?accno=ED510220.

[10]. Kalkofen, D., Sandor, C., White, S. \& Schmalstieg, D. 2011. Visualization Techniques for Augmented Reality. In B. Furht, ed. Handbook of Augmented Reality. New York, NY: Springer New York, pp. 65-98. (en linea) http://www.springerlink.com/index/10.1 007/978-1-4614-0064-6. Acceso: 9 August (2013).

[11]. Krevelen, D.W.F. van \& Poelman, R. 2010. A Survey of Augmented Reality Technologies, Applications and Limitations. The International Journal of Virtual Reality 9(2): 1-20. (en linea) http://kjcomps.6te.net/upload/paper1 .pdf.

[12]. New Media Consortium 2010. Two to Three Years: Simple Augmented Reality. In The Horizon Report 2010. New Media Consortium, pp. 21-24. (en linea) http://www.nmc.org/pdf/2010Horizon-Report.pdf.

[13]. Prensky, M. 2001a. Digital GameBased Learning 1st ed. M. Hill, ed., S. Paul, MN: Mcgraw Hill Book Co. (en linea) http://www.citeulike.org/group/3060/arti cle/2856989. Acceso: 23 November (2012). 
[14]. Prensky, M. 2001b. Digital Natives Digital, Immigrants. On the Horizon 9(8).

[15]. Tobar, H.F., Fabregat, R. \& Baldiris, S. 2013. AR Learning Videogame For Kids With ADHD Symptoms. Universitat de Girona.

[16]. Woods, E., Billinghurst, M., Looser, J., Aldridge, G., Brown, D., Garrie, B. \& Nelles, C. 2004. Augmenting the science centre and museum experience. Proceedings of the 2nd international conference on Computer graphics and interactive techniques in Australasia and South East Asia: 230. (en linea) http://dl.acm.org/citation.cfm?id=98883 4.988873. Acceso: 12 November (2012). 